This article is forthcoming in

The Annals of the American Academy of Political and Social Science

\title{
Nudging social media sharing towards accuracy
}

\author{
Gordon Pennycook ${ }^{1,2 *} \&$ David G. Rand ${ }^{3 * *}$ \\ ${ }^{1}$ Hill/Levene Schools of Business, University of Regina, ${ }^{2}$ Department of Psychology, University of Regina, \\ ${ }^{3}$ Sloan School of Management, Massachusetts Institute of Technology
}

Recent work indicates that a meaningful portion of online misinformation sharing can be attributed to users merely failing to consider accuracy when deciding what to share. As a result, simply redirecting attention to the concept of accuracy can increase sharing discernment. Here we discuss the relevance of accuracy, and outline a limited-attention utility model that formalizes a theory about inattention to accuracy on social media. Research showing how a simple nudge or prompt that shifts attention to accuracy increases the quality of news that people share (typically by decreasing the sharing of false content) is reviewed. We then discuss outstanding questions relating to accuracy nudges, including the need for more work relating to persistence and habituation, as well as a dearth of cross-cultural research. We also make several recommendations for policy makers and social media companies on how to implement accuracy nudges.

Keywords: misinformation, nudges, interventions, social media, inattention

*Corresponding author: gordon.pennycook@uregina.ca, 3737 Wascana Pky, Regina, Saskatchewan, Canada, S4S 0A2, 306-585-4717 
"It's not the tweets, it's the retweets that get you in trouble... You see something that looks good and you don't investigate it."- Donald Trump (Interview with Barstool Sports, 2020)

There is widespread concern about falsehoods spreading on the internet, often via social media. ${ }^{1}$ Here we will review a burgeoning literature on a relatively new approach to the online misinformation problem that has been gaining traction: Accuracy "nudges" (or prompts, or primes) that increase the quality of content that users share on social media. We focus on accuracy nudges because other common types of interventions against misinformation, such as debunking/fact-checking or educational approaches that teach people to identify misinformation, are reviewed extensively elsewhere (Chan et al. 2017; Kozyreva, Lewandowsky, and Hertwig 2020; van der Linden et al. 2021; Traberg, Roozenbeek, and van der Linden 2022). Accuracy nudges also have the advantage of being extremely fast to administer, and not requiring foreknowledge of which news stories are accurate versus misleading. Furthermore, we focus on the sharing of misinformation on social media because it is a context where social science-based interventions may actually be implemented by technologies companies (unlike, say, focusing on foreign influence operations, domestic political elites, established news organizations, or talk radio). We will overview the current state of knowledge, and illuminate what we believe to be the most important next steps for investigating accuracy nudge approaches.

\section{Inattention to accuracy}

There are a multitude of reasons why one might share false or misleading news on social media. Perhaps the content triggers some sort of moral or emotional response (Brady, Crockett, and Van Bavel 2020; Brady et al. 2017), or it could be reaffirming of one's political (or other)

identity (Van Bavel and Pereira 2018), or it could simple seem amusing, interesting or important (Chen, Pennycook, and Rand 2021). However, each of these things are not, of course, unique to 
falsehoods (although in some - perhaps many - cases, inaccurate content may actually be more engaging than accurate content). Thus, rather than asking what causes people to share falsehoods per se, a key question is what factors preferentially cause people to share false news relative to true news - i.e., what influences the overall quality of content that people share? (sometimes referred to as sharing discernment; Pennycook and Rand 2021b).

There are undoubtedly many answers to this question. Here, we focus on one such explanation, namely a lack of attention to accuracy when deciding what to share on social media (Pennycook et al. 2021). By this account, even if people (i) are able to tell truth from falsehood and (ii) have a strong preference not to share inaccurate news (i.e., would not choose to share news they realized to be inaccurate), they may still share misinformation due to inattention. This account is premised on the idea that, due to cognitive constraints, attention is limited when people make sharing decisions. The way in which many people use social media - e.g., scrolling quickly through large numbers of posts, news intermixed with content for which accuracy is not relevant (e.g., family photos, cat videos, etc.), using social media as a way to relax and unwind rather than think critically - is not conducive to thinking carefully about accuracy. Instead, social media focuses users' attention on factors other than accuracy, such as social factors. For example, social media platforms provide immediate, highly quantitative social information about each post (e.g. how many people, and which specific friends, shared and liked each post), which is likely to focus attention on these social cues (and has been shown to affect sharing intentions (Avram et al. 2020). Furthermore, the vocal minority of Americans who are highly partisan may consume a disproportionate amount of airtime on social media, which could cause people to focus more on partisanship (and less on accuracy) than they otherwise would. Thus, people who choose to not share content they realize is inaccurate may never stop to consider the content's 
accuracy in the first place, and thereby wind-up sharing misinformation (thus acting against their actual preference).

\section{Formalizing the inattention-based account of misinformation sharing}

This inattention account was formalized by (Pennycook et al. 2021) using a limitedattention utility model. The modeling framework combines three lines of theory. The first is utility theory, which is the cornerstone of economic models of choice (Stigler 1950; Barberis 2013). When people are choosing across a set of options (in our case, whether or not to share a given piece of content), they preferentially choose the option that gives them more utility, and the utility they gain for a given choice is defined by their preferences. In virtually all such models, preferences are assumed to be fixed (or at least to change over much longer timescales than that of any specific decision, e.g., months or years).

The second line of theorizing involves importance of attention. A core tenet of psychological theory is that when attention is drawn to a particular dimension of the environment (broadly construed), that dimension tends to receive more weight in subsequent decisions (Ajzen 2001; Simon and Newell 1971). While attention has been a primary focus in psychology, it has only recently begun to be integrated with utility theory models - such that attention can increase the weight put on certain preference dimensions over others when making decisions (Bordalo, Gennaioli, and Shleifer 2012; Koszegi and Szeidl 2012).

A third major body of work documents how our cognitive capacities are limited (and our rationality is bounded) such that we are not able to bring all relevant pieces of information to bear on a given decision (Camerer, Loewenstein, and Rabin 2004; Evans and Stanovich 2013; Simon 1972). While the integration of cognitive constraints and utility theory is a core topic in 
behavioral economics, this approach has typically not been applied to attention and the implementation of preferences. Combining these three approaches, Pennycook et al. (2021) presented a model in which attention operates via cognitive constraints: agents are limited to only considering a subset of their preferences in any given decision, and attention determines which preferences are considered. Importantly, therefore, in this model it is attention - and not preferences (or motivations) - that is affected by context/interventions.

Consider a piece of content $x$ which is defined by $k$ different characteristic dimensions; one of these dimensions is whether the content is false/misleading $F(x)$, and the other $k-1$ dimensions are non-accuracy-related (e.g., partisan alignment, humorousness, etc.) defined as $C_{2}(x) \ldots C_{k}(x)$. In our model, the utility a given person expects to derive from sharing content $x$ is given by

$$
U(x)=-a_{1} \beta_{F} F(x)+\sum_{i=2}^{k} a_{i} \beta_{i} C_{i}(x)
$$

where $\beta_{F}$ indicates how much they dislike sharing misleading content and $\beta_{2} \ldots \beta_{k}$ indicate how much they care about each of the other dimensions (i.e., $\beta$ s indicate preferences); while $a_{1}$ indicates how much the person is paying attention to accuracy, and $a_{2} \ldots a_{k}$ indicate how much the person is paying attention to each of the other dimensions. The probability that the person chooses to share the piece of content $x$ is then some increasing function of $U(x)$.

In the standard utility theory model, $a_{i}=1$ for all $i$ (all preferences are considered in every decision). Thus, preference terms with larger $\beta$ values necessarily exert more influence on sharing decisions. The limited-attention model of Pennycook et al. (2021) instead uses the $a_{i}$ values to incorporate cognitive constraints: people can consider only a subset of characteristic dimensions when making decisions. Specifically, agents can only attend to $m$ out of the $k$ utility 
terms in a given decision. That is, each value of $a$ is either 0 or $1, a_{i} \in\{0,1\}$; and because only $m$ terms can be considered at once, the $a$ values must sum to $k, \sum_{i=1}^{k} a_{i}=m$. Critically, the probability that any specific set of preference terms is attended to (i.e., which $a$ values are equal to 1) is heavily influenced by the situation, and (unlike preferences) can change from moment to moment.

Thus, even if people have a strong preference for not sharing inaccurate content (i.e., $\beta_{F}$ is as large, or larger than, other $\beta$ values), how accurate content is may still have little impact on what people decide to share if the context focuses their limited attention on other dimensions. The accuracy-based account of misinformation sharing, then, is the hypothesis that $\beta_{F}$ is not smaller than the other $\beta$ values (e.g., the $\beta$ for political concordance, such that people do not want to share false but politically concordant news) but that people nonetheless sometimes share misinformation because the probability of observing $a_{l}=1$ is far less than $1\left(p\left(a_{l}=1\right)<<1\right)-$ i.e., people often fail to consider accuracy. That is, this account predicts a substantial disconnect between accuracy judgments and sharing intentions, whereby accuracy judgments will be much more sensitive to the veracity of news content than sharing intentions. Furthermore, the inattention-based account predicts that prompts that cause people to attend to accuracy can increase veracity's role in sharing by increasing the probability that $a_{l}=1\left(p\left(a_{l}=1\right) \mid\right.$ treatment $>$ $p\left(a_{l}=1\right) \mid$ control $)$. That is, the accuracy prompt "shines an attentional spotlight" on the accuracy dimension, increasing its chance to influence judgments. Importantly, our theory proposes that accuracy prompts lead people to reallocate their attention to difference preferences (i.e., the $a$ terms), and does not cause changes in one's preferences/processing goal (which are captured by the $\beta$ terms in the model). 


\section{Disconnect between accuracy judgments and sharing intentions}

In support of the model's first prediction, experiments have revealed a strong disconnect between accuracy judgments and sharing intentions in survey experiments. (One cannot easily assess accuracy judgments in observational studies using social media data, making survey experiments a key tool for investigating these questions.) In these experiments, participants are randomized to either rate the accuracy of a series of news posts, or indicate their willingness to share the same series of news posts. When people are asked judge accuracy (and thus necessarily have their attention focused on accuracy), they are often very good at distinguishing between true and false new headlines (e.g., averaging the accuracy ratings of even small groups of laypeople can produce as much agreement with professional fact-checkers as professional factcheckers show with each other; Allen et al. 2020).

However, even in these situations where people who are asked about accuracy can identify news veracity very well, veracity typically has very little impact when people are asked whether they would share the headlines online (sans an accuracy prompt) (Pennycook, McPhetres, et al. 2020; Pennycook et al. 2021; Epstein et al. 2021). Instead, whether the news aligns with the respondents' partisanship is a much stronger predictor of sharing than whether the news is true. This stands in stark contrast to accuracy judgments, where truth is a far stronger predictor than partisan alignment. Thus, a common finding is that the fraction of false headlines selected for sharing is meaningfully larger than the fraction of false headlines that are rated as accurate - implying that people are willing to share headlines that they would be able to identify as being accurate if they thought about it (see also Fazio 2020).

\section{Using accuracy prompts to improve sharing discernment}


The inattention account posits that this disconnect is due to people forgetting to consider accuracy in their sharing decisions. Therefore, shifting people's attention towards the concept of accuracy will cause them to be more discerning in their sharing. An alternative account for this pattern, however, is that people knowingly share false content (e.g., because they have a need for chaos; Arceneaux et al. 2021) - in which case shifting people's attention towards the concept of accuracy will not have a meaningful impact (because they already realize the content is inaccurate, and are choosing to share it anyway).

These competing accounts can be disambiguated using experiments that prime the concept of accuracy. The results of a series of such experiments support the inattention account. For example, if participants are asked rate the accuracy of a single politically neutral news headline at the outset of a survey experiment (an "Evaluation" accuracy prompt treatment, sometimes introduced to participants as part of a pretest for a different study), their subsequent sharing of news relating to politics or COVID-19 (Pennycook, McPhetres, et al. 2020; Epstein et al. 2021; Roozenbeek, Freeman, and van der Linden 2021; Pennycook et al. 2021) becomes more discerning (e.g., people share relatively less false content compared to a control condition where attention is not drawn to accuracy). Fitting the limited-attention utility model to data from such experiments finds that, as per the inattention-based account, the average participant has a strong preference to not share inaccurate news (the $\beta$ on not sharing inaccurate content was as large or larger than the $\beta$ value for sharing politically concordant news) but participants often failed to attend to accuracy ( $a$ value on the accuracy-related preference term was 0 ) leading them to indicate that they would share inaccurate news. Supporting the model's interpretation that the accuracy nudge works by redirecting attention rather than altering motivations/preferences, Pennycook et al. (2021) found that the treatment had no significant effect on responses to a post- 
experimental question asking "How important is it to you that you only share news articles on social media (such as Facebook and Twitter) if they are accurate?".

Critically, the accuracy prompt effect is not driven simply by asking participants any question at the beginning of the experiment: Asking about how funny or entertaining the headlines had no impact on subsequent sharing (i.e., the extent to which people share more true content than false content) (Pennycook et al. 2021). Furthermore, the Evaluation intervention is not the only way to shift attention towards accuracy. Several different types of accuracy prompts have been used, providing support for strong conceptual replicability (Epstein et al. 2021; Pennycook and Rand 2021a). For example, providing minimal digital literacy tips (Epstein et al. 2021) or simply asking participants how important it was to them to share only accurate news (Pennycook et al. 2021; Epstein et al. 2021) increased sharing discernment. Epstein et al. (2021) also found that the effect was particularly strong (i.e., a 100\% increase in sharing discernment) for a more lengthy intervention where participants judged the accuracy of four headlines and were told whether each response was correct to reinforce accuracy. In contrast, simply providing descriptive norm information by telling participants that other people thought it was important to share only accurate news was not particularly effective - perhaps because such interventions also draw attention to social considerations and not just accuracy.

How robust is this accuracy prompt effect? In a recent meta-analysis, we analyzed every experiment we ran $(k=20, N=26,863)$ with accuracy prompts in U.S. samples between 20172020 (Pennycook and Rand 2021a). There are two major drawbacks to typical meta-analyses that this approach overcomes: Publication bias and questionable research practices (and, in particular, p-hacking) (Carter et al. 2019). With respect to publication bias, since our meta-analysis includes the entire "file-drawer", inclusion is not conditioned on the outcome of the study and, hence, 
there is no publication bias. Standard meta-analyses cannot guarantee that publication bias has not occurred because it is not possible to know if all possible experiments have been included (Rothstein, Sutton, and Borenstein 2006). With respect to p-hacking, our meta-analysis uses the exact same analytic procedure across all experiments and this procedure was preregistered in the earliest experiments. Thus, the effect size estimates in individual studies are not influenced by researcher degrees of freedom (Simmons, Nelson, and Simonsohn 2011). Consequently, the effect size estimate produced by the meta-analysis is more credible than typical meta-analyses. We also note that although our meta-analysis only represents the studies from a single research group, we believe that less is gained by independent replications relative to physical lab studies because all studies were run online - it is not clear how much is gained from having an identical study launched by a different group.

The overall effect of accuracy prompts across the 20 experiments was a $72 \%$ increase in sharing discernment relative to control. The effect was stronger for longer interventions, and was not significantly moderated by gender, race, political ideology, education, or the value people explicitly placed on accuracy. (There was some evidence of moderation by political partisanship, such that accuracy prompts were less effective among Republicans despite having an overall positive effect; however, this moderation only occurred robustly for convenience samples recruited from Amazon's Mechanical Turk and not for more representative samples from Lucid and YouGov). We also found that the effect was larger for people who are older, who are higher in cognitive reflection, and who passed more attention check questions (although the effect was observed even among younger adults and those low in cognitive reflection). The central conclusion from these analyses is that, although there is some heterogeneity in the size of the effect, it is quite robust across subgroups. Nonetheless, it is unclear how effective the 
intervention would be against so-called "super-spreaders" (Grinberg et al. 2019; Guess, Nyhan, and Reifler 2020) who share a very large amount of misinformation on social media, since we cannot tell whether these people are represented in our surveys.

One of the key pieces of evidence that accuracy prompts improve sharing discernment by drawing attention to accuracy is that the effect is calibrated to the underlying plausibility of the headlines. That is, it is has been shown consistently that the treatment effect is most strongly negative for headlines that are judged to be the most implausible (based on out-of-sample ratings of headline likelihood) (Pennycook et al. 2021; Epstein et al. 2021; Pennycook, McPhetres, et al. 2020; Roozenbeek, Freeman, and van der Linden 2021). The meta-analysis found a correlation of $r=-.757$ between headline plausibility and the treatment effect across 15 experiments, thus providing strong evidence that accuracy prompts reduce sharing to the extent that headlines are likely to be perceived as inaccurate. This makes sense given the proposed attentional mechanism: An increased focus on accuracy can only impact sharing insofar as headlines are readily discernable as false (or true).

Do accuracy prompt effects generalize from survey experiments to actual behavior on social media? To shed light on this question, Pennycook et al. (2021) conducted a digital field experiment (for a review of this methodology, see Mosleh, Pennycook, and Rand 2021). Specifically, Twitter users who had (re)tweeted articles from Breitbart and Infowars (two highly partisan right-wing websites that produce misleading content) were followed by one of several innocuous "bots" (e.g., 'CookingBot'). The users who followed-back the bot accounts formed the subject pool of the experiment, and where sent a private message asking them to judge the accuracy of a neutral news headline (replicating the Evaluation intervention from the survey experiments). To allow causal inference, a stepped-wedge design was used in which all users 
received the message but were randomly assigned to treatment date. Naturally, very few users responded to the message, but it is not necessary for them respond - simply reading the message is sufficient to be 'treated'. Accordingly, intent-to-treat analyses found that there was a significant increase in the quality of news content that users shared in the 24 hours following the direct message, as measured by the trustworthiness of the news sources that were shared. In addition to demonstrating the practical applicability of the accuracy nudge approach, this field experiment, in which participants did not know they were part of a study, helps to address potential concerns about experimental demand effects/social desirability in the survey experiments. (Also relevant is the observation from the survey experiment of Epstein et al. (2021) that a descriptive social norms treatment - which is likely to invoke social desirability more than the Evaluation treatment - had no effect on sharing discernment.)

\section{Outstanding questions and future directions}

Although there is clear evidence in both survey experiments and a digital field experiment that accuracy prompts can improve the quality of content that people share on social media, there are nonetheless various outstanding questions about the approach that need to be addressed. Most fundamentally, work on accuracy prompts has focused almost entirely on the United States, despite the global nature of online misinformation. Cross-cultural studies are essential for assessing the generalizability of the approach.

Another practical question relates to the time-course of the effect: How quickly does the effect wear off? It is difficult to evaluate this question in survey experiments: although the metaanalysis of Pennycook \& Rand (2021) does not find evidence of the effect decaying over time, experimental sessions are typically not very long. The digital field experiment on Twitter that 
was mentioned earlier recorded tweets in the 24 hours that followed the intervention, but the experiment did not have sufficient power to test for longer effects, and it is not possible to observe when the message was read, making it even harder to assess persistence. From a theoretical perspective, given the fickle nature of attention there is little reason to believe that the interventions would last particularly long. Thus, rather than a one-time treatment to reduce misinformation, the implication of the accuracy prompt studies is that attention matters - and that therefore, technology companies need to make more fundamental changes to the social media ecosystem to systematically redirect attention to accuracy.

Another factor to consider is whether people would become habituated to accuracy prompts, thereby decreasing the impact of the intervention in the long run. A natural solution to this problem is for platforms to use a wide variety of accuracy nudges, constantly rotating which prompts are applied for any given user. Such variation should help to mitigate habituation. It is also possible that accuracy prompts may cause people to adopt the habit of pausing and thinking about accuracy before they share content (related to accuracy) on social media, even absent any reminders. Ultimately, future research is needed to determine the potential downstream consequences of a long-term accuracy prompt campaign. This means that social media companies need to think carefully about, and run experiments to determine, optimal implementation rather than simply deploying the designs used in our studies. Ideally, the results of these experiments would be shared with the public, including investigating potential negative externalities (such as decreasing sharing of accurate news from unknown - and thus distrusted; Pennycook \& Rand, 2019b - sources).

Another important practical question is how accuracy prompts may interact with other approaches to fighting misinformation. One appeal of accuracy prompts relative to most other 
interventions is that accuracy prompts entirely preserve user autonomy, and do not require any centralized definition of truth versus falsehood. Nonetheless, accuracy prompts may interact beneficially with more traditional approaches. One clear point of synergy involves attempts to leverage crowdsourcing to identify misinformation at scale (Epstein, Pennycook, and Rand 2020; Pennycook and Rand 2019b; Allen et al. 2021). Periodically asking users to rate the accuracy of content while they scroll through their newsfeed will not only prompt them to consider accuracy when making subsequent sharing decisions, but can also help generate crowd ratings that platforms can use to help inform ranking algorithms or misinformation labeling. Accuracy prompts are also likely to work in a synergistic fashion with media literacy and other educational or fact-checking interventions aimed at improving users' truth discernment (Guess et al. 2020; Van Der Linden et al. 2021; Nieminen and Rapeli 2018; Traberg et al. 2022). An increased focus on accuracy can only improve sharing insofar as users can successfully assess which headlines are inaccurate - and so improving users' truth discernment will magnify the impact of accuracy prompts. And conversely, increasing users' understanding of what is accurate or inaccurate will not have a strong impact on sharing if they fail to consider accuracy when deciding what to share. Thus, accuracy prompts will magnify the long-term impact of media literacy interventions on subsequent sharing.

One might expect warnings or corrections for misinformation either from fact-checkers, or from other users to also work as accuracy prompts. However, because they are often only applied to a small fraction of inaccurate content (due to scalability issues), fact-checker warnings may unintentionally boost belief in, and sharing of, unlabeled false claims if users infer that the absence of a tag implies verification (the "implied truth effect") (Pennycook, Bear, et al. 2020). And a recent field experiment on social corrections delivered by bots designed to look like other 
users found that social corrections decreased the quality of news that users subsequently

retweeted (Mosleh et al. 2021) - the opposite of what was found using the Evaluation accuracy nudge intervention. Future work is needed to identify what messaging features most effectively redirect attention to accuracy without causing reactance or distraction.

There are also theoretical issues relating to inattention to accuracy and the efficacy of accuracy prompts that should be explored. For example, if people are not paying attention to accuracy when making judgments about what to share, what are they paying attention to? It seems likely that the social signaling and feedback mechanisms that are inherent to most social media applications (such as 'likes') reinforces social considerations, but what precisely are these considerations? Relatedly, it is likely that content that is highly emotional (Martel, Pennycook, and Rand 2020) or that contains moral language (Brady, Crockett, and Van Bavel 2020) will draw people's attention and perhaps undermine their ability, or willingness, to pause and consider the accuracy of the content. Recent work also shows that news headlines that seem to be important or timely are more likely to be shared (and that, with a set of close to 200 headlines, false headlines seemed more important) (Chen, Pennycook, and Rand 2021). Future work should investigate these issues in more detail.

Another theoretical issue pertains to the role of intuitive versus analytic thinking in the sharing of misinformation, and how this intersects with inattention to accuracy. Specifically, it has been shown repeatedly that people who are more reflective and analytic (relative to those who tent to rely on intuitive gut feelings) are better able to distinguish between true and false news (Pennycook and Rand 2019c, 2019a) (for a meta-analysis see Pennycook and Rand, 2021c), and that people are more likely to believe false news (but not true news) when they are forced to rely on their intuition (Bago, Rand, and Pennycook 2020) or are instructed to rely on 
emotion (Martel, Pennycook, and Rand 2020). Furthermore, there is evidence that people who are more reflective are also more discerning in what they share on social media, both in survey studies (Pennycook and Rand 2021a) and on Twitter (Mosleh, Pennycook, et al. 2021) (although see Osmundsen et al., 2021, who find no relationship between cognitive reflection and fake news sharing on Twitter). Furthermore, people who are more reflective are also more strongly impacted by the accuracy prompt intervention (as noted above) (Epstein et al. 2021; Pennycook and Rand 2021a). It seems plausible, then, that part of the reason why people are inattentive to accuracy is because they are not engaging sufficient reflection on social media. Alternatively, however, it could be that the accuracy prompts do not induce more reflection, but merely change what factors people are reflecting on. Future empirical and theoretical work is needed to distinguish between these possibilities.

\section{Conclusion}

We argue that at least some sharing of misinformation on social media can be attributed to users merely failing to consider accuracy when deciding what to share and have outlined a limited-attention utility model that formalizes our theoretical perspective on this issue. Consistent with this model, experimental evidence consistently indicates that simply redirecting attention to the concept of accuracy can increase sharing discernment. There are, nonetheless, several outstanding questions relating to accuracy nudges that we surfaced here. We hope this present review will serve as a useful guide for future research, as well as for policy makers and social media companies who may wish to implement accuracy nudges. 


\section{Acknowledgments}

We gratefully acknowledge the financial support of the William and Flora Hewlett Foundation, the Omidyar Network, the John Templeton Foundation, the Social Sciences and Humanities Research Council of Canada, the Canadian Institutes of Health Research, and the Miami Foundation.

\section{References}

Ajzen, Icek. 2001. "Nature and Operation of Attitudes." Annual Review of Psychology 52 (1): 27-58. https://doi.org/10.1146/annurev.psych.52.1.27.

Allen, Jennifer, Antonio Alonso Arechar, G. Pennycook, and David G. Rand. 2021. "Scaling up Fact-Checking Using the Wisdom of Crowds." Science Advances. https://psyarxiv.com/9qdza/.

Arceneaux, Kevin, Timothy B. Gravelle, Matthias Osmundsen, Michael Bang Petersen, Jason Reifler, and Thomas J. Scotto. 2021. "Some People Just Want to Watch the World Burn: The Prevalence, Psychology and Politics of the "Need for Chaos." Philosophical Transactions of the Royal Society B: Biological Sciences 376 (1822). https://doi.org/10.1098/rstb.2020.0147.

Avram, Mihai, Nicholas Micallef, Sameer Patil, and Filippo Menczer. 2020. "Exposure to Social Engagement Metrics Increases Vulnerability to Misinformation." Harvard Kennedy School Misinformation Review, July. https://doi.org/10.37016/mr-2020-033.

Bago, B., D.G Rand, and G. Pennycook. 2020. "Fake News, Fast and Slow: Deliberation Reduces Belief in False (but Not True) News Headlines." Journal of Experimental Psychology: General 149 (8). https://doi.org/10.31234/OSF.IO/29B4J.

Barberis, Nicholas C. 2013. "Thirty Years of Prospect Theory in Economics: A Review and 
Assessment." Journal of Economic Perspectives. https://doi.org/10.1257/jep.27.1.173.

Bavel, J. J. Van, and A. Pereira. 2018. “The Partisan Brain: An Identity-Based Model of Political Belief." Trends in Cognitive Sciences.

Bordalo, P., N. Gennaioli, and A. Shleifer. 2012. "Salience Theory of Choice Under Risk." The Quarterly Journal of Economics 127 (3): 1243-85. https://doi.org/10.1093/QJE.

Brady, William J., Molly J. Crockett, and J. J. Van Bavel. 2020. “The MAD Model of Moral Contagion: The Role of Motivation, Attention and Design in the Spread of Moralized Content Online." Perspectives on Psychological Science. https://doi.org/10.31234/OSF.IO/PZ9G6.

Brady, William J., Julian A. Wills, John T. Jost, Joshua A. Tucker, and Jay J. Van Bavel. 2017. "Emotion Shapes the Diffusion of Moralized Content in Social Networks." Proceedings of the National Academy of Sciences 114 (28): 7313-18. https://doi.org/10.1073/pnas.1618923114.

Camerer, Colin F, George Loewenstein, and Matthew Rabin. 2004. Advances in Behavioral Economics. Princeton: Princeton University Press.

Carter, Evan C., Felix D. Schönbrodt, Will M. Gervais, and Joseph Hilgard. 2019. “Correcting for Bias in Psychology: A Comparison of Meta-Analytic Methods:" Https://Doi.Org/10.1177/2515245919847196 2 (2): 115-44. https://doi.org/10.1177/2515245919847196.

Chan, Man pui Sally, Christopher R. Jones, Kathleen Hall Jamieson, and Dolores Albarracín. 2017. "Debunking: A Meta-Analysis of the Psychological Efficacy of Messages Countering Misinformation." Psychological Science 28 (11): 1531-46. https://doi.org/10.1177/0956797617714579. 
Chen, Cathy Xi, Gordon Pennycook, and David Rand. 2021. "What Makes News Sharable on Social Media?" PsyArXiv. https://doi.org/10.31234/OSF.IO/GZQCD.

Epstein, Ziv, Adam J. Berinsky, Rocky Cole, Andrew Gully, Gordon Pennycook, and David G. Rand. 2021. "Developing an Accuracy-Prompt Toolkit to Reduce COVID-19 Misinformation Online." Harvard Kennedy School Misinformation Review, May. https://doi.org/10.37016/mr-2020-71.

Epstein, Ziv, G. Pennycook, and D. G. Rand. 2020. "Will the Crowd Game the Algorithm?” In Proceedings of the 2020 CHI Conference on Human Factors in Computing Systems, 1-11. New York, NY, USA: Association for Computing Machinery (ACM). https://doi.org/10.1145/3313831.3376232.

Evans, J. St. B. T., and K. E. Stanovich. 2013. "Dual-Process Theories of Higher Cognition: Advancing the Debate." Perspectives on Psychological Science 8 (3): 223-41. https://doi.org/10.1177/1745691612460685.

Fazio, Lisa. 2020. "Pausing to Consider Why a Headline Is True or False Can Help Reduce the Sharing of False News." Harvard Kennedy School Misinformation Review 1 (2). https://doi.org/10.37016/mr-2020-009.

Grinberg, N., K. Joseph, L. Friedland, B. Swire-Thompson, and D. Lazer. 2019. “Fake News on Twitter during the 2016 U.S. Presidential Election." Science 363: 374-78.

Guess, A. M., Michael Lerner, Benjamin Lyons, Jacob M. Montgomery, Brendan Nyhan, Jason Reifler, and Neelanjan Sircar. 2020. “A Digital Media Literacy Intervention Increases Discernment between Mainstream and False News in the United States and India." Proceedings of the National Academy of Sciences, June, 201920498. https://doi.org/10.1073/pnas.1920498117. 
Guess, A. M., Brendan Nyhan, and Jason Reifler. 2020. "Exposure to Untrustworthy Websites in the 2016 US Election." Nature Human Behaviour 4 (5): 472-80. https://doi.org/10.1038/s41562-020-0833-X.

Koszegi, B., and A. Szeidl. 2012. "A Model of Focusing in Economic Choice.” The Quarterly Journal of Economics 128 (1): 53-104. https://doi.org/10.1093/QJE.

Kozyreva, A., S. Lewandowsky, and R. Hertwig. 2020. “Citizens Versus the Internet: Confronting Digital Challenges With Cognitive Tools.” Psychological Science in the Public Interest 21: 103-56. https://doi.org/10.1177/1529100620946707.

Linden, Sander van der, Jon Roozenbeek, Rakoen Maertens, Melisa Basol, Ondřej Kácha, Steve Rathje, and Cecilie Steenbuch Traberg. 2021. "How Can Psychological Science Help Counter the Spread of Fake News?” Spanish Journal of Psychology. Cambridge University Press. https://doi.org/10.1017/SJP.2021.23.

Martel, C., G. Pennycook, and D.G. Rand. 2020. "Reliance on Emotion Promotes Belief in Fake News." Cognitive Research: Principles and Implications 5 (1). https://doi.org/10.1186/s41235-020-00252-3.

Mosleh, Mohsen, C. Martel, Dean Eckles, and David G. Rand. 2021. "Perverse Consequences of Debunking in a Twitter Field Experiment: Being Corrected for Posting False News Increases Subsequent Sharing of Low Quality, Partisan, and Toxic Content.” ACM Conference on Human Factors in Computing Systems (CHI '21).

Mosleh, Mohsen, G. Pennycook, and D. G. Rand. 2021. "Field Experiments on Social Media." PsyArXiv. https://doi.org/10.31234/OSF.IO/DGMC2.

Mosleh, Mohsen, Gordon Pennycook, Antonio Alonso Arechar, and David Rand. 2021. “Cognitive Reflection Correlates with Behavior on Twitter.” Nature Communications, 1- 
20. https://doi.org/10.31234/OSF.IO/QASWN.

Nieminen, Sakari, and Lauri Rapeli. 2018. "Fighting Misperceptions and Doubting Journalists' Objectivity: A Review of Fact-Checking Literature.” Political Studies Review, July, 147892991878685. https://doi.org/10.1177/1478929918786852.

Osmundsen, Mathias, Alexander Bor, Peter Bjerregaard Vahlstrup, Anja Bechmann, and Michael Bang Petersen. 2021. "Partisan Polarization Is the Primary Psychological Motivation behind 'Fake News' Sharing on Twitter." American Political Science Review. https://doi.org/10.31234/osf.io/v45bk.

Pennycook, G., A. Bear, E. Collins, and D. G. Rand. 2020. “The Implied Truth Effect: Attaching Warnings to a Subset of Fake News Stories Increases Perceived Accuracy of Stories Without Warnings." Management Science, December. https://doi.org/10.1287/mnsc.2019.3478.

Pennycook, G., Ziv Epstein, Mohsen Mosleh, Antonio Alonso Arechar, Dean Eckles, and David Rand. 2021. "Shifting Attention to Accuracy Can Reduce Misinformation Online." Nature, 1-63. https://doi.org/10.1038/s41586-021-03344-2.

Pennycook, G., Jonathon McPhetres, Yunhao Zhang, J. G. Lu, and David G. Rand. 2020. "Fighting COVID-19 Misinformation on Social Media: Experimental Evidence for a Scalable Accuracy Nudge Intervention." Psychological Science. https://doi.org/10.31234/OSF.IO/UHBK9.

Pennycook, G., and D. G. Rand. 2019a. "Who Falls for Fake News? The Roles of Bullshit Receptivity, Overclaiming, Familiarity, and Analytic Thinking." Journal of Personality, August. https://doi.org/10.2139/ssrn.3023545.

—. 2021a. "Reducing the Spread of Fake News by Shifting Attention to Accuracy: Meta- 
Analytic Evidence of Replicability and Generalizability.” PsyArXiv, 1-24. https://doi.org/10.31234/OSF.IO/V8RUJ.

—. 2021b. "Lack of Partisan Bias in the Identification of Fake (versus Real) News." Trends in Cognitive Sciences 25 (9): 725-26. https://doi.org/10.1016/J.TICS.2021.06.003.

Pennycook, G., and D.G. Rand. 2019b. "Fighting Misinformation on Social Media Using Crowdsourced Judgments of News Source Quality.” Proceedings of the National Academy of Sciences of the United States of America 116 (7). https://doi.org/10.1073/pnas.1806781116.

—. 2019c. "Lazy, Not Biased: Susceptibility to Partisan Fake News Is Better Explained by Lack of Reasoning than by Motivated Reasoning." Cognition 188 (April): 39-50. https://doi.org/10.1016/j.cognition.2018.06.011.

Pennycook, G., and David G. Rand. 2021c. “The Psychology of Fake News.” Trends in Cognitive Sciences, 1-29. https://doi.org/10.1016/j.tics.2021.02.007.

Roozenbeek, J., A. F. Freeman, and Sander van der Linden. 2021. "How Accurate Are Accuracy Nudge Interventions? A Pre-Registered Direct Replication of Pennycook et Al. (2020).” Psychological Science.

Rothstein, H. R., A. J. Sutton, and M. Borenstein. 2006. Publication Bias in Meta-Analysis: Prevention, Assessment and Adjustments. Chichester, UK: John Wiley \& Sons, Ltd. Simmons, Joseph P., Leif D. Nelson, and Uri Simonsohn. 2011. "False-Positive Psychology: Undisclosed Flexibility in Data Collection and Analysis Allows Presenting Anything as Significant.” Psychological Science 22 (11): 1359-66.

https://doi.org/10.1177/0956797611417632.

Simon, Herbert A. 1972. "Theories of Bounded Rationality.” In Decision and Organization, 
161-76.

Simon, Herbert A., and Allen Newell. 1971. "Human Problem Solving: The State of the Theory in 1970." American Psychologist 26 (2): 145-59. https://doi.org/10.1037/h0030806.

Stigler, George J. 1950. “The Development of Utility Theory. I.” Journal of Political Economy 58 (4): 307-27. https://doi.org/10.1086/256962.

Traberg, Cecilie Steenbuch, J. Roozenbeek, and Sander van der Linden. 2022. "Psychological Inoculation against Misinformation: Current Evidence and Future Directions.” The Annals of the American Academy of Political and Social Science.

\footnotetext{
${ }^{1}$ Researchers may disagree on the scope of the problem, a debate we will not rehash here.
} 